Originally published as:

Kohler, S., Kleiser, C., Richter, A., Stahl, A., Vohmann, C., Heseker, H., Mensink, G. B. M.,"The Fluid intake of adolescents in Germany. Results collected in EsKiMo ",2007," Ernährung Wissenschaft und Praxis", 1, 10, 444-450

DOI: $10.1007 / s 12082-007-0111-6$

The original publication is available at www.springerlink.com 


\title{
Trinkverhalten von Jugendlichen in Deutschland
}

\author{
Ergebnisse aus EsKiMo
}

\author{
S. Kohler ${ }^{1}$, C. Kleiser ${ }^{1}$, A. Richter ${ }^{1}$, A. Stahl ${ }^{2}$, C. Vohmann ${ }^{2}$, H. Heseker ${ }^{2}$, G.B.M. Mensink ${ }^{1}$ \\ ${ }^{1}$ Abteilung für Epidemiologie und Gesundheitsberichterstattung, Robert Koch-Institut, Berlin \\ ${ }^{2}$ Ernährung und Verbraucherbildung, Department Sport und Ernährung, Fakultät für Naturwissenschaften, Universität \\ Paderborn, Paderborn
}

\begin{abstract}
Studienergebnissen zufolge haben sich die durchschnittlichen Trinkmengen von Kindern und Jugendlichen in Deutschland in den letzten Jahren zwar erhöht, aber die meisten trinken weniger als empfohlen wird [17]. Außerdem wird der Durst oft mit den „falschen“ Getränken gelöscht [5, 12, 25]. $\mathrm{Ob}$ dies für die Kinder und Jugendlichen in Deutschland zutrifft, wurde unter anderem in EsKiMo (Ernährungsstudie als KiGGS-Modul) untersucht. Ergebnisse zum Getränkekonsum der 12-bis 17Jährigen werden im Folgenden vorgestellt.
\end{abstract}

\section{Einleitung}

Getränke sind ein wichtiger Bestandteil der Ernährung. Mit ihnen werden Wasser, Mikro- und Makronährstoffe sowie andere bioaktive Substanzen aufgenommen. Für den Menschen ist Wasser essenziell. Eine ausgeglichene Flüssigkeitsbilanz ist Voraussetzung für den optimalen Ablauf lebensnotwendiger Stoffwechselprozesse und muss bei Flüssigkeitsverlusten durch entsprechende Zufuhr wiederhergestellt werden.

Die für Deutschland gültigen D-A-CH-Richtwerte zur Wasserzufuhr durch Getränke und feste Nahrung für Kinder und Jugendliche sind in . Tab. 1 zusammengefasst [8]. Dabei muss berücksichtigt werden, dass der individuelle Bedarf auch von Lebenssituation, Jahreszeit und körperlicher Aktivität abhängig ist.

Das Forschungsinstitut für Kinderernährung (FKE) gibt im Rahmen der optimiX-Empfehlungen (Konzept der Optimierten Mischkost für eine gesunde Ernährung von Kindern und Jugendlichen) etwas geringere Aufnahmemengen an [11], was darin begründet ist, dass Milch in optimiX getrennt aufgeführt wird (. Tab. 2).

Die Richtwerte für die Zufuhr von Wasser liegen für Jugendliche teilweise (DGE-Richtwerte für die 15bis unter 19-Jährigen) über denen für Erwachsene. Gründe dafür sind einerseits ein größeres Verhältnis von Körperoberfläche zu Körpergewicht, wodurch es zu überproportionalen Flüssigkeitsverlusten über die Hautoberfläche kommen kann, andererseits ein größerer prozentualer Wasseranteil im Körper und ein stoffwechselbedingt höherer Flüssigkeitsbedarf.

Entspricht die zugeführte Flüssigkeitsmenge nicht dem Bedarf, kommt es zur Abnahme des Körperwassers, d. h. zu einer Dehydratation. Diese macht sich durch Durst bemerkbar. Besonders von Senioren, Kindern und Jugendlichen wird dieses Signal jedoch häufig nicht wahrgenommen, was dann eine negative Flüssigkeitsbilanz zur Folge hat. Durch Dehydratation können $u$. a. Konzentrationsstörungen und Leistungsschwäche auftreten [18]. Außerdem kann eine zu geringe Wasserzufuhr zur Entstehung einer Obstipation beitragen [2]. In jüngerem Alter kann eine Dehydratation besonders gravierende gesundheitliche Konsequenzen haben, weil der kindliche Organismus gegenüber dem erwachsenen ein schlechteres Adaptionsvermögen an hitzebedingte Wasserverluste hat [1].

Neben der aufgenommenen Menge spielt auch die Qualität der Getränke eine bedeutende Rolle. Durch Innovationen und ein ständig wachsendes und differenzierteres Angebot eröffnen sich vielfältige Möglichkeiten zur Deckung des Flüssigkeitsbedarfs. Gesundheitliche Probleme wie Karies [23], Übergewicht $[21,22,27]$ und Alkoholismus [14] sind unter Umständen mit einem zu hohen Konsum bestimmter Getränkearten assoziiert. 
Tab. 1 Richtwerte für die Zufuhr von Wasser durch Getränke und feste Nahrung in ml/Tag [8]

\begin{tabular}{lll} 
Alter & $\begin{array}{l}\text { Wasserzufuhr durch Getränke } \\
\text { (ml/Tag) }\end{array}$ & $\begin{array}{l}\text { Wasserzufuhr durch feste } \\
\text { Nahrung (ml/Tag) }\end{array}$ \\
\hline 10 bis unter 13 Jahre & 1170 & 710 \\
\hline 13 bis unter 15 Jahre & 1330 & 810 \\
\hline 15 bis unter 19 Jahre & 1530 & 920
\end{tabular}

Tab. 2 Altersgemäße Getränkemengen in der Optimierten Mischkost in ml/Tag [11] (Stand: März 2007)

\begin{tabular}{lcc} 
Alter & $\begin{array}{l}\text { Empfohlene Getränkemenge Empfohlene Getränkemenge } \\
\text { (ml/Tag); Mädchen } \\
\text { (ml/Tag); Jungen }\end{array}$ \\
\hline 10 bis 12 Jahre & 1000 & 1000 \\
\hline 13 bis 14 Jahre & 1200 & 1300 \\
\hline 15 bis 18 Jahre & 1400 & 1500
\end{tabular}

Tab. 3 Tägliche Verzehrsmengen von Getränken bei den 12- bis 17-Jährigen (in ml)

\begin{tabular}{|c|c|c|c|c|c|c|c|c|c|c|c|}
\hline \multirow[t]{2}{*}{ Getränkesorte } & & \multicolumn{5}{|c|}{ Jungen } & \multicolumn{5}{|c|}{ Mädchen } \\
\hline & & $\overline{\mathrm{x}}$ & $s$ & Median & P10 & P90 & $\overline{\mathrm{x}}$ & $s$ & Median & P10 & P90 \\
\hline \multirow{10}{*}{$\begin{array}{l}\text { Alkoholfreie } \\
\text { Getränke }\end{array}$} & Gesamt & 2061 & 1028 & 1809 & 977 & 3333 & 1835 & 896 & 1601 & 888 & 2987 \\
\hline & - Wasser & 1011 & 964 & 750 & 29 & 2291 & 959 & 797 & 770 & 90 & 2013 \\
\hline & - Kaffee & 24 & 97 & 0 & 0 & 43 & 28 & 92 & 0 & 0 & 72 \\
\hline & - Tee & 102 & 261 & 0 & 0 & 320 & 143 & 295 & 21 & 0 & 429 \\
\hline & - Saft/Saftgetränke & 395 & 454 & 257 & 0 & 946 & 384 & 430 & 257 & 0 & 870 \\
\hline & - Softdrinks & 529 & 739 & 250 & 0 & 1406 & 320 & 535 & 86 & 0 & 1012 \\
\hline & - Limonade & 360 & 582 & 125 & 0 & 1129 & 196 & 381 & 47 & 0 & 601 \\
\hline & davon zuckerhaltige Limonade & 315 & 517 & 100 & 0 & 1001 & 160 & 346 & 30 & 0 & 504 \\
\hline & davon kalorienreduzierte Limonade & 45 & 277 & 0 & 0 & 36 & 36 & 163 & 0 & 0 & 48 \\
\hline & - Andere Softdrinks & 169 & 402 & 0 & 0 & 500 & 123 & 348 & 0 & 0 & 375 \\
\hline \multirow{5}{*}{$\begin{array}{l}\text { Alkoholische } \\
\text { Getränke }\end{array}$} & Gesamt & 102 & 236 & 0 & 0 & 314 & 38 & 96 & 0 & 0 & 136 \\
\hline & - Bier & 88 & 207 & 0 & 0 & 286 & 25 & 74 & 0 & 0 & 71 \\
\hline & - Wein & 3 & 14 & 0 & 0 & 3 & 4 & 15 & 0 & 0 & 10 \\
\hline & - Spirituosen etc. & 11 & 47 & 0 & 0 & 21 & 9 & 39 & 0 & 0 & 17 \\
\hline & - Alkopops & 6 & 36 & 0 & 0 & 0 & 5 & 30 & 0 & 0 & 0 \\
\hline
\end{tabular}

$\overline{\mathrm{X}}$ : Mittelwert, s: Standardabweichung, P10: 10. Perzentile, P90: 90. Perzentile

\section{Methoden}

EsKiMo ist eine Nachfolgeerhebung von KiGGS (Kinder- und Jugendgesundheitssurvey) und hat das Ernährungsverhalten von insgesamt 2506 Kindern und Jugendlichen im Alter von 6 bis 17 Jahren im gesamten Bundesgebiet von Januar bis Dezember 2006 erfasst. Die Eltern der teilnehmenden Kinder unter 12 Jahren haben gemeinsam mit ihrem Kind ein 3-Tage-Verzehrsprotokoll geführt. Die 12- bis 17-Jährigen wurden mit Hilfe des Ernährungserhebungsprogramms DISHES (Dietary Interview Software for Health Examination Studies) persönlich zu ihrer Ernährung in den letzten 4 Wochen befragt $[3,24]$. So wurden neben den Daten über die verzehrten Lebensmittel detaillierte Informationen zum Trinkverhalten und zum Getränkekonsum von Kindern und Jugendlichen gewonnen und anschließend analysiert. An dieser Stelle werden Ergebnisse für die 1272 Teilnehmer von Beginn des 12. bis einschließlich des 17. Lebensjahrs dargestellt. Teilweise werden dabei die Daten für die 12- bis 13-Jährigen, die 14- bis 15-Jährigen und die 16- bis 17-Jährigen gemeinsam ausgewertet. 


\section{Ergebnisse}

Einen Überblick über den täglichen Getränkekonsum der 12- bis 17-Jährigen gibt . Tab. 3. Angegeben sind sowohl die Mittelwerte als auch die Mediane. Für die weitere Auswertung werden grundsätzlich die Mediane verwendet, bei Alkohol, kalorienreduzierten Limonaden, Tee, Kaffee und der Gruppe „andere Softdrinks“ wegen der geringen Anzahl der Konsumenten die Mittelwerte. Der Konsum von Milch wird bei dieser Auswertung nicht berücksichtigt.
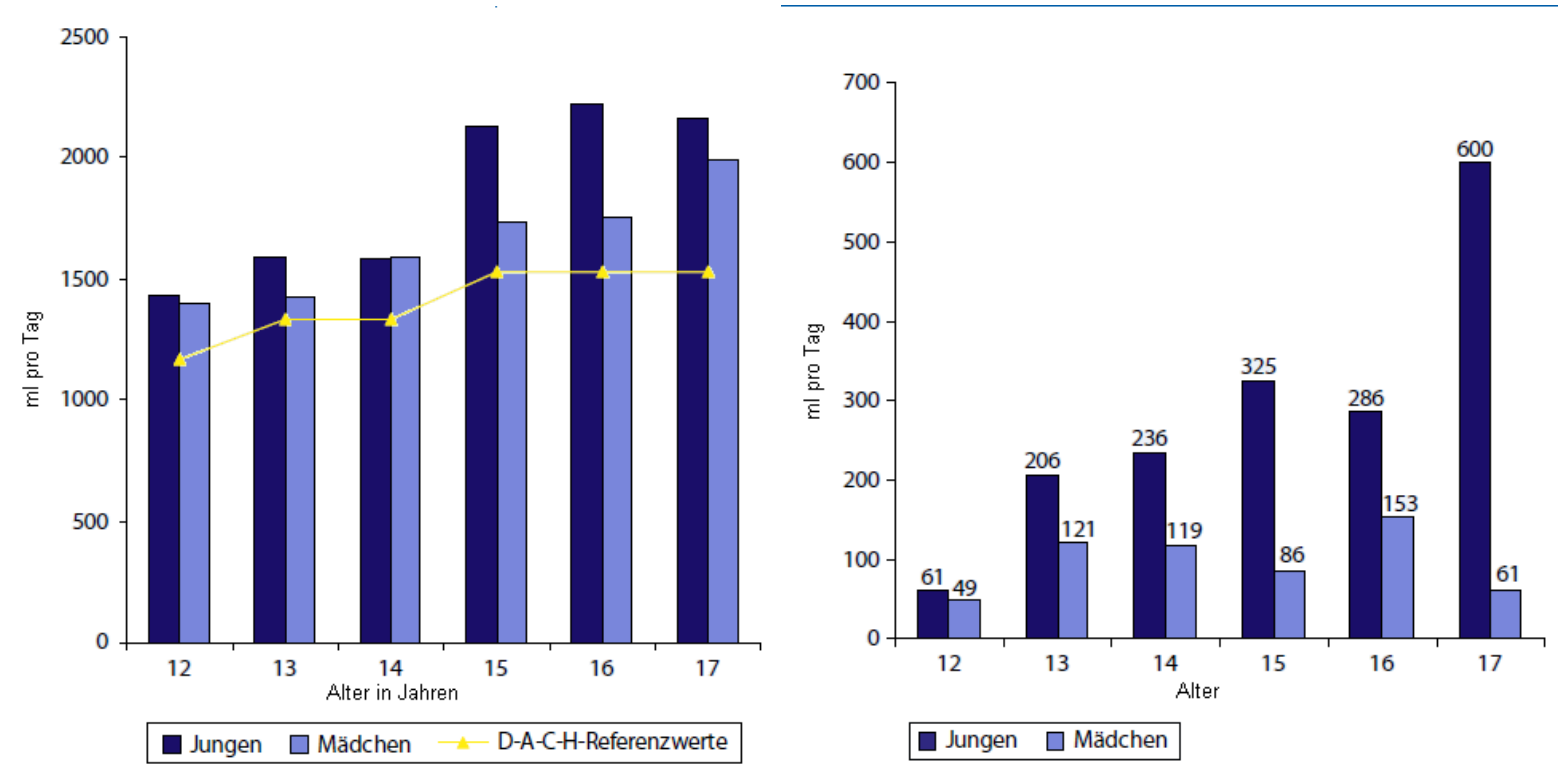

Abb. 1 Konsum alkoholfreier Getränke (Median und D-A-CH Referenzwerte in ml/Tag) Abb. 2 Konsum von Softdrinks (Median in $\mathrm{ml} / \mathrm{Tag}$ )

\section{Alkoholfreie Getränke}

Die Gruppe der alkoholfreien Getränke umfasst Wasser, Saft/Saftgetränke, Softdrinks, Kaffee und Tee. Zu den Softdrinks zählen Limonaden, kalorienreduzierte Limonaden und die „anderen Softdrinks“. In der Kategorie „andere Softdrinks“ sind Eistee, Energiedrinks, isotonische Getränke, Malzbier bzW. alkoholfreies Bier, Wellnessgetränke, aromatisiertes Wasser und Frühstücksdrinks zusammengefasst, die nur in sehr geringen Mengen verzehrt wurden.

Die tägliche mediane Verzehrsmenge an alkoholfreien Getränken beträgt $1809 \mathrm{ml}$ bei den Jungen und $1609 \mathrm{ml}$ bei den Mädchen. Insgesamt steigt die Trinkmenge mit zunehmendem Alter. Die . Abb. 1 zeigt, dass nur geringe Unterschiede bei Jungen und Mädchen im Alter zwischen 12 und 14 Jahren bestehen. Ein Anstieg der Trinkmenge ist bei den Jungen ab 15 Jahren zu erkennen. Die höchsten Mengen an alkoholfreien Getränken konsumieren die 16-jährigen Jungen mit einer medianen Menge von $2223 \mathrm{ml}$ pro Tag. Bei den Mädchen ist der Konsum in der Gruppe der 17-Jährigen am höchsten.

Die tägliche Energiezufuhr durch alkoholfreie Getränke beträgt bei den Jungen durchschnittlich 379 kcal und bei den Mädchen 288 kcal. Dies entspricht 12,3\% bzw. 12,5\% der gesamten Energiezufuhr. Aus alkoholischen Getränken stammen im Mittel bei den Jungen 1,7\% und bei den Mädchen 1,0\% der täglichen Energiezufuhr. Saft/Saftgetränke und Softdrinks sind die Hauptenergiequellen unter den Getränken. Während die Mädchen durch Saft/Saftgetränke fast doppelt soviel Kalorien aufnehmen wie durch Softdrinks, tragen bei den Jungen die Softdrinks etwas stärker als Saft/Saftgetränke zur Energiezufuhr bei. Die Beliebtheit von Wasser, Saft/Saftgetränken und Softdrinks wird aus . Tab. 3 ersichtlich. 


\section{Wasser}

Unter der Kategorie Wasser ist in diesem Zusammenhang industriell aufbereitetes Mineral-, Quell-, Heil- oder Tafelwasser, aber auch Trinkwasser zu verstehen. Durch Suppen und feste Speisen aufgenommenes Wasser wird in diesem Fall nicht dazugezählt.

Alle teilnehmenden Jugendlichen haben angegeben, während der erfragten 4 Wochen Wasser getrunken zu haben. Die durchschnittliche Zufuhr liegt bei $754 \mathrm{ml}$ täglich. Somit ist Wasser das meistgetrunkene Getränk.

Die Zufuhrmenge unterscheidet sich zwischen den Geschlechtern nur gering (. Tab. 3). Durchschnittlich verzehren die Mädchen $20 \mathrm{ml}$ mehr als die Jungen. Mit $552 \mathrm{ml}$ täglich trinken die 14 Jahre alten männlichen Teilnehmer am wenigsten, dagegen konsumieren die 16-jährigen Jungen mit $1000 \mathrm{ml}$ beinahe doppelt so viel. Bei den Mädchen bestehen geringere Unterschiede zwischen den Altersgruppen.

\section{Saft/Saftgetränke}

Zur Gruppe der Säfte zählen Obst- und Gemüsesäfte, Fruchtnektare, Fruchtsaftgetränke und Fruchtsaftschorlen.

Etwa 98\% der Jugendlichen haben im Erhebungszeitraum Saft/Saftgetränke getrunken. Durchschnittlich beträgt die tägliche Zufuhr $257 \mathrm{ml}$ und ist für beide Geschlechter gleich hoch. Zwischen den Altersgruppen sind keine eindeutigen Unterschiede erkennbar. Pro Tag trinken die 12bis 13-jährigen Jungen und Mädchen im Durchschnitt $260 \mathrm{ml}$, die 14- bis 15-Jährigen $283 \mathrm{ml}$ und die 16- bis 17-Jährigen $231 \mathrm{ml}$ Saft/Saftgetränke. Unter allen Teilnehmern sind die höchsten täglichen Verzehrsmengen bei den 15-jährigen Jungen mit $362 \mathrm{ml}$ und die niedrigsten bei den 16-jährigen Mädchen mit $205 \mathrm{ml}$ zu verzeichnen.

Die weiblichen Teilnehmer nehmen $185 \mathrm{kcal}$ pro Tag durch Saft/Saftgetränke auf. Bei den männlichen Teilnehmern liegt die tägliche Energiezufuhr durch Saft/Saftgetränke bei 188 kcal.

\section{Softdrinks}

Zur Gruppe der Softdrinks zählen die Untergruppen Limonaden (gezuckerte und Light-Produkte) und "andere Softdrinks".

Laut den Befragungsergebnissen trinken 84\% aller Jugendlichen Softdrinks. Bei den Jungen liegt der Anteil der Konsumenten mit 88\% leicht über dem der Mädchen mit 80\%. Jungen trinken in allen Altersklassen mengenmäßig mehr Softdrinks als Mädchen - mit durchschnittlich 250 ml pro Tag beinahe dreimal soviel wie die weiblichen Teilnehmer mit $86 \mathrm{ml}$. Die Zufuhrmenge steigt bei den männlichen Jugendlichen anders als bei den weiblichen mit dem Alter deutlich an. Die 17 Jahre alten Jungen trinken mit $600 \mathrm{ml}$ pro Tag am meisten, die 12 Jahre alten Mädchen mit $49 \mathrm{ml}$ pro Tag am wenigsten (. Abb. 2). Die tägliche Energiezufuhr durch Softdrinks insgesamt liegt bei den männlichen Teilnehmern mit 189 kcal über der der Mädchen mit 101 kcal.

Limonade trinken 78\% aller Jugendlichen. Der Anteil der männlichen Limonadentrinker liegt mit 82\% um 8\% über dem der Mädchen. Limonade ist mit einer medianen Verzehrsmenge von $125 \mathrm{ml}$ am Tag bei den Jungen und $47 \mathrm{ml}$ bei den Mädchen die beliebteste Softdrinkvariante. Mit einer Tageszufuhr von $379 \mathrm{ml}$ konsumieren die 17-jährigen Jungen am meisten und übertreffen die Mengen der gleichaltrigen Mädchen um über das Zehnfache. Von allen Teilnehmern haben die 12-jährigen Jungen und Mädchen am wenigsten Limonade getrunken. Des Weiteren zeigen die Zahlen, dass bei den Jungen mit zunehmendem Alter der Limonadenkonsum fast kontinuierlich steigt, bei den Mädchen ist dies nicht der Fall. Während die männlichen Teilnehmer durch Limonade im Mittel täglich 156 kcal aufnehmen, sind es bei den weiblichen Teilnehmern nur $79 \mathrm{kcal}$.

Vorwiegend trinken die Jugendlichen gezuckerte Limonaden. Während des Befragungszeitraums von 4 Wochen haben 79\% aller Jungen gezuckerte Limonaden getrunken, bei den Mädchen ist der Anteil mit $67 \%$ geringer. Wieder verzehren die männlichen Teilnehmer mit einer dreifach höheren Menge pro Tag deutlich mehr als die weiblichen (. Tab. 3). Am meisten trinken die 17-jährigen Jungen mit täglich $286 \mathrm{ml}$ gezuckerte Limonaden. Während die Tagesmengen der 12- bis 13-jährigen Jungen im Durchschnitt $24 \mathrm{ml}$ betragen, liegen die Werte der 14- bis 15-jährigen Jungen bei $73 \mathrm{ml}$ und die der 16- bis 17-jährigen bei $171 \mathrm{ml}$. Bei den Mädchen nimmt die Gruppe der 12- bis 13-Jährigen $21 \mathrm{ml}$ auf, die der 14- bis 15-Jährigen $43 \mathrm{ml}$ und die der 16- bis 17-Jährigen $25 \mathrm{ml}$. Durch zuckerhaltige Limonaden werden bei den männlichen Teilnehmern täglich $154 \mathrm{kcal}$ und bei den weiblichen Teilnehmern 77 kcal zugeführt. 
Im Befragungszeitraum haben 13\% der Jugendlichen - 12\% der Jungen und 15\% der Mädchen kalorienreduzierte Limonade getrunken. Im Folgenden werden aufgrund der geringen Anzahl der Konsumenten die Mittelwerte betrachtet. Die mittlere tägliche Aufnahme energiereduzierter Limonade beträgt $45 \mathrm{ml}$ bei den Jungen und $36 \mathrm{ml}$ bei den Mädchen. Beim Altersverlauf zeigt sich, dass die 16jährigen Jugendlichen mit $65 \mathrm{ml}$ am Tag am meisten, die 12 Jahre alten Teilnehmer mit $15 \mathrm{ml}$ dagegen am wenigsten energiereduzierte Limonaden trinken.

Die Kategorie „andere Softdrinks“ umfasst nichtalkoholische Getränke wie Eistee, aromatisiertes Wasser, Malzgetränke, Wellness-, Fitness- und Frühstücksdrinks. Solche Getränke haben $43 \%$ aller Teilnehmer zu sich genommen. Den Studienergebnissen zufolge werden sie eher von Jungen als von Mädchen getrunken. Im Mittel konsumieren die männlichen Teilnehmer pro Tag 169 ml, die weiblichen Teilnehmer 123 ml Getränke aus der Kategorie „andere Softdrinks“.

\section{Tee}

Tee (schwarzer/grüner Tee und/oder Früchte- oder Kräutertee) wird von 48\% der Teilnehmer getrunken. Mit einer mittleren täglichen Trinkmenge von $102 \mathrm{ml}$ bei den Jungen und $143 \mathrm{ml}$ bei den Mädchen wird Tee von Jugendlichen vor Kaffee als weiterem Heißgetränk bevorzugt. Die mittleren Mengen an Kräuter- oder Früchtetee sind dreimal höher als die an schwarzem oder grünem Tee. Mädchen trinken insgesamt mehr Tee als Jungen. Altersbedingte Unterschiede sind nicht zu erkennen.

\section{Kaffee}

Zur Kategorie Kaffee gehören in dieser Auswertung sämtliche Kalt- oder Heißgetränke, die Kaffeeanteile beinhalten. Der Milchanteil in den Milchkaffeegetränken findet jedoch keine Berücksichtigung.

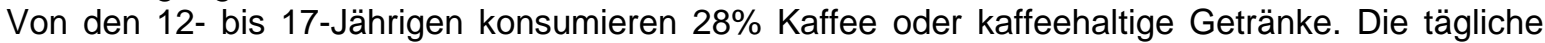
mittlere Aufnahme liegt bei den Mädchen mit $28 \mathrm{ml}$ leicht über dem der Jungen mit $24 \mathrm{ml}$. Während die Jüngeren keinen bzw. kaum Kaffee trinken, steigt der Verzehr mit zunehmendem Alter an.

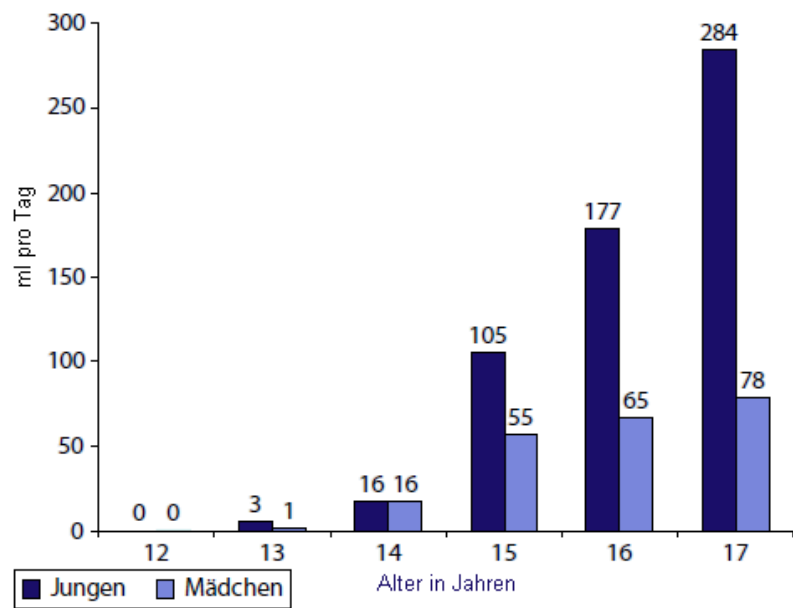

Abb. 3 Konsum alkoholischer Getränke (Mittelwert in $\mathrm{ml} / \mathrm{Tag}$ ) 


\section{Alkoholische Getränke}

In der vorliegenden Auswertung werden die alkoholischen Getränke in drei Gruppen aufgegliedert:F Bier, inklusive Biermischgetränke,F Sekt, Wein, weinhaltige Getränke,F Spirituosen etc. (einschließlich Likör bzw. Likörwein, Branntwein, Cocktails und Alkopops).

Aufgrund der politischen Debatte und dem dadurch resultierenden öffentlichen Interesse werden Alkopops getrennt betrachtet. Unter Alkopops sind im Rahmen dieser Auswertung wein- oder spirituosenhaltige Limonaden, die zudem Taurin, Guarana oder Koffein beinhalten können, zu verstehen. Während der erfragten vier Wochen haben 54\% aller Jungen und 51\% der Mädchen mindestens einmal alkoholhaltige Getränke getrunken. Unter den EsKiMo-Teilnehmern ist der Alkoholkonsum bei Jungen höher als bei Mädchen (. Abb. 3). Mit zunehmendem Alter erhöhen sich bei beiden Geschlechtern die aufgenommenen Mengen alkoholischer Getränke. Während die 12- bis 13-Jährigen kaum Alkohol trinken, liegt die mittlere tägliche Trinkmenge (sämtliche alkoholische Getränke) bei den 14- bis 15-jährigen Jungen bei $10 \mathrm{ml}$ und bei den Mädchen bei $9 \mathrm{ml}$. Zu einem deutlichen Anstieg des Konsums kommt es im Alter von 16-17 Jahren. Die Mädchen dieser Altersgruppe trinken im Mittel täglich $66 \mathrm{ml}$ alkoholische Getränke, die Jungen mit $189 \mathrm{ml}$ deutlich mehr.

Insgesamt $76 \%$ der 16- bis 17 -jährigen Jungen und $71 \%$ der gleichaltrigen Mädchen geben an, alkoholische Getränke zu konsumieren. Innerhalb des Befragungszeitraums von vier Wochen haben $34 \%$ der Teilnehmer mindestens einmal Bier getrunken. Vorrangig wird dieses von den Jungen konsumiert: 15-jährige Jungen bzw. Mädchen trinken pro Tag im Mittel $97 \mathrm{ml}$ bzw. $41 \mathrm{ml}$, die 16jährigen $152 \mathrm{ml}$ bzw. $46 \mathrm{ml}$ und die 17-jährigen $244 \mathrm{ml} \mathrm{bzw.} 43 \mathrm{ml}$. Werden ausschließlich die männlichen Jugendlichen, die Bier trinken, betrachtet, ergeben sich mittlere tägliche Aufnahmen von $206 \mathrm{ml}$ bei den 15-Jährigen, $199 \mathrm{ml}$ bei den 16-Jährigen und $310 \mathrm{ml}$ bei den 17-Jährigen.

Von den Teilnehmern haben 33\% im Befragungszeitraum Spirituosen etc. getrunken.

Spirituosen (einschließlich Likör bzw. Likörwein, Branntwein, Cocktails und Alkopops) werden hauptsächlich von den älteren Jugendlichen getrunken. Die tägliche mittlere Menge beträgt bei den 16- bis 17-jährigen Jungen $20 \mathrm{ml}$, bei den Mädchen $16 \mathrm{ml}$.

Werden allein die Trinkmengen der Konsumenten von Spirituosen betrachtet, sind es im Mittel bei den Jungen und Mädchen im Alter von 16 bis 17 Jahren pro Tag $44 \mathrm{ml} \mathrm{bzw.} 33 \mathrm{ml}$. Alkopops haben 7\% aller Jugendlichen getrunken. Im täglichen Mittel werden von denjenigen Jungen und Mädchen, die Alkopops trinken, $87 \mathrm{ml} \mathrm{bzw.} 70 \mathrm{ml}$ konsumiert.

Wein oder weinhaltige Getränke haben 19\% der Teilnehmer getrunken. Diese trinken im Mittel $18 \mathrm{ml}$ pro Tag, was pro Woche in etwa einem Weinglas mit $125 \mathrm{ml}$ entspricht.

Im Rahmen der weiteren Auswertung wurden auch die aufgenommenen Alkoholmengen in Gramm mittels BLS II.3 berechnet [19]. Die im Mittel zugeführten Mengen liegen bei den 17-jährigen Jungen mit $13 \mathrm{~g}$ reinem Alkohol pro Tag deutlich über den $4 \mathrm{~g}$ der gleichaltrigen Mädchen sowie der 16jährigen Jungen und Mädchen mit $8 \mathrm{~g}$ bzw. $3 \mathrm{~g}$.

Die durch den Bierkonsum täglich zugeführte Alkoholmenge beträgt bei den 17 Jahre alten Jungen 8 $\mathrm{g}$, gefolgt von den 16-jährigen Jungen mit $5 \mathrm{~g}$. Mädchen konsumieren den meisten Alkohol über Spirituosen etc., dennoch liegen die dadurch zugeführten reinen Alkoholmengen der 17-jährigen mit $3 \mathrm{~g}$ sowie die der 16-jährigen mit $1 \mathrm{~g}$ noch unter den $4 \mathrm{~g}$ der 17-jährigen Jungen. Die Alkoholaufnahme durch Alkopops ist gering.

\section{Diskussion}

Die hier dargestellten Ergebnisse vermitteln einen aktuellen, repräsentativen Überblick über den Getränkekonsum von Jugendlichen in Deutschland.

Die Daten von EsKiMo zeigen, dass die Mehrheit der 12- bis 17-Jährigen ihren Flüssigkeitsbedarf deckt. Während in der KiGGS-Studie sowie in weiteren Untersuchungen ein starker Anstieg beim Softdrinkkonsum und die Verdrängung anderer Getränken beobachtet wurden [5, 12, 25], konsumierten die Teilnehmer von EsKiMo im Durchschnitt ausreichend Wasser. Mineral- bzw. Leitungswasser sind besonders gut zur Deckung des Flüssigkeitsbedarfs geeignet und bei den EsKiMo-Teilnehmern das einzige Getränk, das ausnahmslos alle getrunken haben.

Saft/Saftgetränke werden von den Mädchen und jüngeren Jungen mengenmäßig mehr als Softdrinks aufgenommen. Dies entspricht den Beobachtungen in der DONALD-Studie über den Getränkekonsum von Kindern und Jugendlichen [26].

Während die Zufuhr von Saft/Saftgetränken bei Mädchen und Jungen gleich ist, konsumieren die männlichen Teilnehmer (vor allem die über 15-Jährigen) deutlich mehr Softdrinks. Eine Studie der North American Association for the Study of Obesity zeigte ebenfalls diese Tendenz [28]. Allgemein betrachtet muss ein erhöhter Verzehr von gezuckerten Softdrinks kritisch bewertet werden. Kinder, die 
bevorzugt Softdrinks zu sich nehmen, trinken weniger Saft und Milch [15, 27]. Marshall et al. [23] konnten außerdem einen vermehrten Kariesbefall durch häufigen Verzehr von Softdrinks feststellen. Des Weiteren sind Zusammenhänge zwischen dem erhöhten Konsum von gezuckerten Softdrinks und der Entstehung von Adipositas wahrscheinlich [15, 21, 22]. So führt die zusätzliche Aufnahme von Energie beim Verzehr zuckerreicher Getränke offenbar nicht zu einer Reduktion der sonstigen Nahrungszufuhr. Auch haben flüssige Lebensmittel insgesamt eine geringere Sättigungswirkung als feste Lebensmittel $[10,27]$. Bei erwachsenen Personen mit regelmäßigem Konsum von Softdrinks wurde ein signifikant erhöhtes Risiko für die Entwicklung einer Adipositas und eines metabolischen Syndroms beobachtet [9]. Die bisher vorliegenden Untersuchungsergebnisse werden als ausreichend angesehen, eine Reduktion des Softdrinkkonsums zu empfehlen [7, 13]. International gibt es derzeit bereits viele Kampagnen zur Reduzierung eines übermäßigen Softdrinkkonsums, beispielsweise mit Hilfe von Aufklärungsplakaten und Angaben zu den jeweiligen Energiegehalten der einzelnen Getränke [4]. In Großbritannien, Frankreich und einigen US-amerikanischen Städten wurden daher Softdrinks bereits an den Schulen verboten [27].

Allerdings lässt sich daraus nicht folgern, dass die verzehrten Limonadenmengen zwangsläufig zu einer Erhöhung der Energieaufnahme führen. Ein hoher Gesamtlimonadenkonsum ist noch kein eindeutiges Indiz für eine hohe Energiezufuhr, weil durch die zahlreichen Light-Produkte auch energiearme Limonaden getrunken werden. Daher wurde für die Erhebungen im Rahmen von EsKiMo zwischen gezuckerten und kalorienreduzierten Limonaden unterschieden. Bei genauer Differenzierung der einzelnen Softdrinkarten wird allerdings deutlich, dass Diätlimonaden bei Jugendlichen nur eine sehr geringe Bedeutung haben. Die Ergebnisse einer US-amerikanischen Studie, wonach bei Mädchen mit zunehmendem Alter der Konsum von Light-Produkten steigt [5], konnte weder für die weiblichen noch für die männlichen Teilnehmer von EsKiMo bestätigt werden.

Der Konsum von Heißgetränken ist laut EsKiMo-Daten mengenmäßig weniger bedeutend. Die generelle Beliebtheit von Kaffee vor Tee bei Erwachsenen ist nach diesen Auswertungen unter den Jugendlichen nicht zu erkennen. Wenn überhaupt Heißgetränke getrunken werden, dann sind es hauptsächlich Früchte- oder Kräutertees.

Im Gegensatz zu den bisher umstrittenen gesundheitlichen Auswirkungen mancher nichtalkoholischer Getränke ist das hohe Suchtpotenzial alkoholischer Getränke erwiesen [14]. Dem Konsum alkoholischer Getränke kommt somit eine besondere Bedeutung zu und er sollte besonders kritisch betrachtet werden.

Ergebnisse einer europäischen Schülerstudie von 2003 zeigen, dass circa 85\% der 15- bis 16jährigen Jugendlichen bereits einmal Alkohol konsumiert haben. Neben Bier spielten Alkopops eine erhebliche Rolle [20]. In einer aktuellen Umfrage der DAK (Deutsche Angestellten-Krankenkasse) gaben ein Viertel von ungefähr 1600 befragten Schülern der 7. Klassenstufe an, in den letzten 4 Wochen Alkohol getrunken zu haben [16]. Laut einer Repräsentativbefragung der Bundeszentrale für gesundheitliche Aufklärung (BZgA) zum Alkoholkonsum in Deutschland in den Jahren 2004-2007 haben 49\% der 12- bis 17-jährigen Jugendlichen mindestens einmal im Monat Alkohol getrunken [6]. Mit einem Anteil von 54\% der männlichen und 51\% der weiblichen Befragten, die während des Zeitraums von 4 Wochen mindestens einmal Alkohol getrunken haben, kommen die Ergebnisse von EsKiMo denen der BZgA sehr nahe.

Die aktuellen Debatten über den Alkoholkonsum bei Jugendlichen beziehen sich vor allem auf das Trinken bis zum extremen Rauschzustand. Bei näherer Betrachtung der EsKiMo-Zahlen konsumieren vor allem die 16- (Mittelwert: $177 \mathrm{ml} / \mathrm{Tag}$ ) und 17-jährigen (Mittelwert: $284 \mathrm{ml} / \mathrm{Tag}$ ) männlichen Teilnehmer alkoholische Getränke. Nach der Repräsentativbefragung der BZgA trinken Jungen im Alter von 16 bis 17 Jahren pro Woche $154 \mathrm{~g}$ reinen Alkohol. Diese Befragung deckt zusätzlich auf, dass nach einer Abnahme vom Jahr 2004 zu 2005 die wöchentlich konsumierte Alkoholmenge in den letzten zwei Jahren wieder gestiegen ist. Werden zum Vergleich die täglich zugeführten Alkoholmengen der männlichen EsKiMo-Befragten auf eine Woche umgerechnet, sind die Mengen mit $56 \mathrm{~g}$ bei den 16-jährigen bzw. $91 \mathrm{~g}$ bei den 17-jährigen Jungen deutlich niedriger. Diese Unterschiede sind möglicherweise durch unterschiedliche Befragungsmethoden, Under- bzw. Overreporting oder durch andere Ursachen mitbedingt. Auch wenn es gewisse Differenzen zwischen den zwei Studien gibt, so belegen beide einen auffälligen Bierkonsum männlicher Jugendlicher. Zahlen der europäischen Schülerstudie zu Alkohol und anderen Drogen (ESPAD) bestätigen dies [20]. Sowohl bei der BZgAals auch der EsKiMo-Erhebung lässt sich die Alkoholaufnahme der männlichen Jugendlichen im Alter von 16 und 17 Jahren vorrangig durch deren Bierkonsum erklären. Die damit aufgenommene Menge Alkohol beträgt laut BZgA $90 \mathrm{~g}$ pro Woche. Die 16 bzw. 17 Jahre alten männlichen EsKiMo-Befragten liegen mit $35 \mathrm{~g}$ bzw. $57 \mathrm{~g}$ Alkohol aus Bier pro Woche zwar darunter; die Mengen sind aber trotzdem nicht als niedrig zu bewerten. Der mittlere Konsum der 16- und 17-jährigen männlichen Biertrinker von EsKiMo entspricht einer Menge von 1391 bzw. 2173 ml Bier wöchentlich, bei den gleichaltrigen, Bier trinkenden Mädchen sind es 643 bzw. 666 ml pro Woche. 
Der in den vergangenen Jahren diskutierte Trend zu hohem Konsum von Alkopops kann anhand der EsKiMo-Daten nicht mehr festgestellt werden. Generell sind Alkopops Limonadenmischgetränke, die Spirituosen enthalten. Durch öffentlichen Druck und zum Schutz von Kindern und Jugendlichen wurde die Abgabe an diese verboten und die Hersteller wurden verpflichtet, Alkopops mit dem Hinweis „Abgabe an Personen unter 18 Jahren verboten“ zu kennzeichnen. Des Weiteren wird auf branntweinhaltige Süßgetränke seit 2004 eine Sondersteuer erhoben. Ziel dieser Maßnahmen war es, einen Rückgang des Konsums unter Kindern und Jugendlichen zu bewirken. Inzwischen haben die Hersteller zusätzlich Alkopops entwickelt, die statt Spirituosen Bier oder Wein enthalten, daher nicht unter die Sondersteuer fallen und auch an 16-Jährige verkauft werden können. Für junge Menschen stellen Alkopops eine besondere Gefahr dar, weil sie aufgrund ihres Limonadenanteils süß bzw. fruchtig schmecken, so den typischen Alkoholgeschmack überdecken und dadurch verführen, mehr zu trinken. Bei genauerer Analyse der Trinkmuster der EsKiMo-Teilnehmer sind Alkopops zwar bei den Jungen etwas beliebter als Wein, nicht aber bei den Mädchen. Darüber hinaus ist der Konsum relativ gering: Nur 7\% der konsumierten alkoholischen Getränken sind Alkopops. Der Rückgang des Konsums ist möglicherweise auf die erwähnte Verordnung zurückzuführen.

\section{Fazit}

Die EsKiMo-Daten zeigen, dass die meisten Jugendlichen ihren Flüssigkeitsbedarf ausreichend decken. Dieses positive Ergebnis steht im Kontrast zu Daten früherer Erhebungen und kann eventuell als ein Teilerfolg bisheriger Kampagnen zur Verbesserung eines gesunden Ernährungsverhaltens gewertet werden sowie deren Nutzen bestätigen. Neben einer ausreichenden Flüssigkeitszufuhr ist die ernährungsphysiologische Qualität der konsumierten Getränke von entscheidender Bedeutung. Hier wird bei der Auswertung deutlich, dass Softdrinks und Bier hauptsächlich von 16- und 17-jährigen Jungen in unerwünscht hohem Maße getrunken werden.

\section{Literatur}

1. Anonymus (2000) Climatic heat stress and the exercising child and adolescent. American Academy of Pediatrics. Committee on Sports Medicine and Fitness. Pediatrics 106: 158-159

2. Arnaud MJ (2003) Mild dehydration: a risk factor of constipation? Eur J Clin Nutr 57 [Suppl 2]: S88-95

3. Bauch A, Mensink GBM, Vohmann C et al. (2006) EsKiMo. Die Ernährungsstudie bei Kindern und Jugendlichen. ErnährungsUmschau 53: 380-385

4. Bergen D, Yeh MC (2006) Effects of energy-content labels and motivational posters on sales of sugar-sweetened beverages: stimulating sales of diet drinks among adults study. J Am Diet Assoc 106: 1866-1869

5. Bowman S (2002) Beverage choices of young females: changes and impact on nutrient intakes. Journal of the American Dietetic Association 102 (9): 1234-1239

6. Bundeszentrale für gesundheitliche Aufklärung (Hrsg) (2007) Alkoholkonsum der Jugendlichen in Deutschland 2004 bis 2007. Ergebnisse der Repräsentativbefragungen der Bundeszentrale für gesundheitliche Aufklärung, Köln

7. DellaValle DM, Roe LS, Rolls BJ (2005) Does the consumption of caloric and non-caloric beverages with a meal affect energy intake? Appetite 44: 187-193

8. DGE, ÖGE, SGE/SVE (Hrsg) (2000) D-A-CH Referenzwerte für die Nährstoffzufuhr. 1. Aufl., Umschau Braus, Frankfurt Wien Basel

9. Dhingra R, Sullivan L, Jacques PF et al. (2007) Soft drink consumption and risk of developing cardiometabolic risk factors and the metabolic syndrome in middle-aged adults in the community. Circulation 116: 480-488

10. Flood JE, Roe LS, Rolls BJ (2006) The effect of increased beverage portion size on energy intake at a meal. J Am Diet Assoc 106: 1984-1990; discussion: 1990-1981

11. Forschungsinstitut für Kinderernährung (FKE) (Hrsg) (2005) Empfehlungen für die Ernährung von Kindern und Jugendlichen - optimiX. 5. überarbeitete Aufl., Dortmund

12. French SA, Lin BH, Guthrie JF (2003) National trends in soft drink consumption among children and adolescents age 6 to 17 years: prevalence, amounts, and sources, 1977/1978 to 1994/1998. J Am Diet Assoc 103: 1326-1331

13. Gibson S, Neate D (2007) Sugar intake, soft drink consumption and body weight among British children: further analysis of National Diet and Nutrition Survey data with adjustment for under-reporting and physical activity. International Journal of Food Sciences and Nutrition 58: 445-460

14. Hahn A, Ströhle A, Wolters M (2005) Ernährung - Physiologische Grundlagen, Prävention, Therapie. Wissenschaftliche Verlagsgesellschaft, Stuttgart

15. Harnack L, Stang J, Story M (1999) Soft drink consumption among US children and adolescents: nutritional consequences. J Am Diet Assoc 99: 436-441

16. Isensee B, Wiborg G, Hanewinkel R (2007) Alkoholkonsum im Jugendalter. Ergebnisse einer Befragung von Schülerinnen und Schülern der 7. Klassenstufe. Institut für Therapie- und Gesundheitsforschung (IFT-Nord), Kiel

17. Kersting M, Alexy U (2003) Fakten zur Kinderernährung. Hans Marseille, München

18. Kleiner SM (1999) Water: an essential but overlooked nutrient. J Am Diet Assoc 99: 200-206

19. Klemm C, Mathis G, Christ M et al. (1999) Der Bundeslebensmittelschlüssel (BLS II.3). Bundesinstitut für gesundheitlichen Verbraucherschutz und Veterinärmedizin, Berlin 
20. Kraus L, Heppekausen K, Barrera A, Orth B (2004) Die Europäische Schülerstudie zu Alkohol und anderen Drogen (ESPAD): Befragung von Schülerinnen und Schülern der 9. und 10. Klasse in Bayern, Berlin, Brandenburg, Hessen, Mecklenburg-Vorpommern und Thüringen. Institut für Therapieforschung, München. IFT-Berichte 141

21. Ludwig DS, Peterson KE, Gortmaker SL (2001) Relation between consumption of sugar-sweetened drinks and childhood obesity: a prospective, oberservational analysis. Lancet 357: 505-508

22. Malik VS, Schulze MB, Hu FB (2006) Intake of sugar-sweetened beverages and weight gain: a systematic review. Am J Clin Nutr 84: 274-288

23. Marshall TA, Levy SM, Broffitt B et al. (2003) Dental caries and beverage consumption in young children. Pediatrics 112: 184-191

24. Mensink GBM, Bauch A, Vohmann C et al. (2007) EsKiMo. Das Ernährungsmodul des Kinder- und Jugendgesundeitssurveys (KiGGS). Ernährung 1: 225-229

25. Mensink GBM, Kleiser C (2006) Informationen für die Teilnehmer der KiGGS-Studie. Bundesgesundheitsbl

Gesundheitsforsch Gesundheitsschutz 49: 1225-1232

26. Sichert-Hellert W, Kersting M (2004) Home-made carbonated water and the consumption of water and other beverages in children and adolescents: results of the DONALD study. Acta Paediatr 93: 1583-1587

27. Vartanian LR, Schwartz MB, Brownell KD (2007) Effects of soft drink consumption on nutrition and health: a systematic review and meta-analysis. Am J Public Health 97: 667-675

28. West DS, Bursac Z, Quimby D et al. (2006) Self-reported sugar-sweetened beverage intake among college students. Obesity (Silver Spring) 14: 1825-1831 